\title{
Expression of the CopB Outer Membrane Protein by Moraxella catarrhalis Is Regulated by Iron and Affects Iron Acquisition from Transferrin and Lactoferrin
}

\author{
CHRISTOPH AEBI, ${ }^{1,2}$ BARBARA STONE,${ }^{3}$ MARGARET BEUCHER,${ }^{3}$ LESLIE D. COPE, ${ }^{1}$ \\ ISOBEL MACIVER, ${ }^{1}$ SHARON E. THOMAS, ${ }^{1}$ GEORGE H. MCCRACKEN, JR., ${ }^{2}$ \\ P. FREDERICK SPARLING, ${ }^{3}$ AND ERIC J. HANSEN ${ }^{1 *}$ \\ Departments of Microbiology ${ }^{1}$ and Pediatrics, ${ }^{2}$ University of Texas Southwestern Medical Center, \\ Dallas, Texas 75235, and Department of Medicine, School of Medicine, University of \\ North Carolina at Chapel Hill, Chapel Hill, North Carolina $27599^{3}$
}

Received 5 January 1996/Accepted 19 February 1996

\begin{abstract}
The amino acid sequence of the cell-surface-exposed, 81-kDa CopB outer membrane protein of Moraxella catarrhalis was found to be similar to those of TonB-dependent outer membrane proteins of other gramnegative bacteria. Expression of CopB was affected by the availability of iron in the growth medium, and the extent of overexpression of $\mathrm{CopB}$ in response to iron limitation varied widely among the $M$. catarrhalis strains tested. Wild-type $M$. catarrhalis strains were found to be able to utilize ferric citrate, transferrin, lactoferrin, and heme as sources of iron for growth in vitro. However, an isogenic copB mutant was severely impaired in its ability to utilize transferrin and lactoferrin as sole sources of iron for growth, whereas this same mutant grew similarly to the wild-type parent strain when supplied with ferric citrate as the iron source. The $\operatorname{cop} B$ mutant was not significantly different from its wild-type parent strain in its ability to bind transferrin and lactoferrin. In addition, the wild-type parent strain and the $\operatorname{cop} B$ mutant exhibited equivalent rates of uptake of ${ }^{55} \mathrm{Fe}$ from ferric citrate. However, the $\operatorname{cop} B$ mutant was markedly less able than the wild-type strain to take up ${ }^{55} \mathrm{Fe}$ from transferrin and lactoferrin. These results indicate that lack of expression of the CopB protein exerts a direct or indirect effect on the ability of $M$. catarrhalis to utilize iron bound to certain carrier proteins.
\end{abstract}

Moraxella (Branhamella) catarrhalis is a common respiratory tract pathogen in humans. In children, this gram-negative organism is responsible for up to $20 \%$ of cases of acute otitis media $(3,14,18)$. In fact, in a recent report, $M$. catarrhalis DNA could be detected by PCR in middle ear effusions from $46 \%$ of patients with chronic otitis media with effusion (40). In adults, $M$. catarrhalis commonly causes lower respiratory tract infections in patients with chronic obstructive lung disease (13, 20,31). In immunocompromised hosts, and mainly in patients with hematologic malignancies, $M$. catarrhalis has been identified as an etiologic agent of bacteremia, meningitis, skeletal infections, endocarditis, and other invasive focal infections (13, $15,20,29,31)$.

The recent recognition of $M$. catarrhalis as an important pathogen in both the upper and lower respiratory tract has resulted in increased interest in its interactions with the human host and, more specifically, in its antigenic composition. Outer membrane proteins constitute major antigenic determinants of this unencapsulated organism (4), and different strains share remarkably similar outer membrane protein profiles $(4,33)$. CopB (outer membrane protein B2), a major and antigenically well-conserved $81-\mathrm{kDa}$ outer membrane protein of $M$. catarrhalis $035 \mathrm{E}$, has been characterized in detail (23). Antibody to this surface-exposed protein is bactericidal and has been shown to enhance the rate of clearance of $M$. catarrhalis from the lungs of mice (23). A copB mutation rendered $M$. catarrhalis less able to resist pulmonary clearance in this mouse model

\footnotetext{
* Corresponding author. Mailing address: Department of Microbiology, University of Texas Southwestern Medical Center, 6000 Harry Hines Blvd., Dallas, TX 75235-9048. Phone: (214) 648-5974. Fax: (214) 648-5905. Electronic mail address: hansen01@utsw.swmed.edu.
}

and also converted the serum-resistant wild-type strain to a serum-sensitive phenotype (24).

Expression of CopB by $M$. catarrhalis strains growing in a rich, undefined medium appeared to be constitutive in a previous study from this laboratory (23). However, a recent report from Campagnari et al. (10) suggested that a number of $M$. catarrhalis outer membrane proteins with apparent molecular masses of 80 to $115 \mathrm{kDa}$, including what appeared to be CopB, were expressed in greater abundance when the availability of iron in the growth medium was limited. In the present study, we show that there are strain-dependent differences in $M$. catarrhalis involving the level of CopB expression in response to iron limitation. Moreover, we have found that the amino acid sequence of the CopB protein resembles that of TonBdependent outer membrane proteins, and especially that of one particular iron-regulated protein from Neisseria gonorrhoeae (6) and Neisseria meningitidis (39). Finally, we have found that an isogenic $c o p B$ mutant is impaired in its ability to utilize both transferrin and lactoferrin as sources of iron for growth in vitro.

\section{MATERIALS AND METHODS}

Bacterial strains and culture conditions. The bacterial strains used in this study are listed in Table 1 . The wild-type $M$. catarrhalis strain $035 \mathrm{E}$ and its isogenic $\operatorname{cop} B$ mutant $035 \mathrm{E}-\mathrm{CopB}^{-}$have been described in detail elsewhere (23, 24). M. catarrhalis $012 \mathrm{E}$ is a serum-resistant isolate derived from middle ear fluid (23). A third wild-type $M$. catarrhalis strain (ATCC 25240) was obtained from the American Type Culture Collection, Rockville, Md. M. catarrhalis strains were routinely cultured in brain heart infusion (BHI) broth (Difco Laboratories, Detroit, Mich.) at $37^{\circ} \mathrm{C}$ or on BHI agar plates in an atmosphere of $95 \%$ air-5\% $\mathrm{CO}_{2}$. BHI broth and agar plates were iron depleted by adding deferoxamine mesylate (Desferal; Ciba-Geigy, Basel, Switzerland) to a final concentration of $30 \mu \mathrm{M}$ (BHI-DF30) and incubating at $4^{\circ} \mathrm{C}$ overnight before use. For ${ }^{55} \mathrm{Fe}$ uptake studies, bacterial cells were grown in chemically defined medium (CDM) as described previously (46). N. gonorrhoeae strains (32) were routinely grown on chocolate agar plates. Haemophilus influenzae type b strain DL42 was grown 
TABLE 1. Bacterial strains used in this study

\begin{tabular}{lll}
\hline \multicolumn{1}{c}{ Strain } & \multicolumn{1}{c}{ Description } & \multicolumn{1}{c}{ Source or reference } \\
\hline $\begin{array}{l}\text { Moraxella catarrhalis } \\
\text { 035E }\end{array}$ & Wild-type isolate from middle ear fluid & 23,24 \\
035E-CopB ${ }^{-}$ & $\begin{array}{l}\text { Isogenic } \operatorname{copB} \text { mutant constructed by electroporating 035E with } \\
\text { a mutated } \operatorname{copB} \text { gene containing a mini-Tn10kan transposon }\end{array}$ & 24 \\
insertion & Wild-type isolate from middle ear fluid & 24 \\
012E & Wild-type isolate & American Type Culture Collection \\
ATCC 25240 & Wild-type isolate & 32 \\
Neisseria gonorrhoeae & lbpA::Tn3(Cm); mutant unable to express lactoferrin-binding & G. Biswas and P. F. Sparling \\
FA19 & protein A & 12 \\
FA6775 & tbpA::mTn3(Cm); mutant unable to express transferrin-binding & 12 \\
FA6747 & protein 1 & 11 \\
Haemophilus influenzae type b DL42 & Wild-type isolate & 42 \\
Escherichia coli HB101 & Cloning host strain &
\end{tabular}

routinely by using BHI medium supplemented with Levinthal's base (1). Escherichia coli $\mathrm{HB} 101$ was grown in Luria-Bertani medium $(30)$ at $37^{\circ} \mathrm{C}$.

Iron sources. Human transferrin ( $30 \%$ iron saturated), human lactoferrin ( $90 \%$ iron saturated), bovine hemin (heme), and human hemoglobin were obtained from Sigma Chemical Co., St. Louis, Mo. Heme:hemopexin complexes and hemoglobin:haptoglobin complexes were provided by Ursula Muller-Eberhard, Cornell Medical Center, New York, N.Y. Ferric citrate was prepared by adding a 10-fold molar excess of sodium citrate to $\mathrm{FeCl}_{3}$.

Growth experiments. Strains $035 \mathrm{E}$ and $035 \mathrm{E}-\mathrm{CopB}^{-}$were grown overnight in BHI broth with aeration. A $100-\mu l$ portion of this overnight culture was inoculated into $10 \mathrm{ml}$ of prewarmed BHI-DF30 in a 500-ml sidearm flask, and cell densities were adjusted to a reading of 35 Klett units with a Klett colorimeter (Klett Manufacturing Co., New York, N.Y.). Cultures were then allowed to grow to late logarithmic phase, yielding a reading of 200 Klett units. This culture was diluted to $10^{-5}$ in prewarmed BHI broth, yielding a suspension containing $10^{4}$ $\mathrm{CFU} / \mathrm{ml}$. Portions $(100 \mu \mathrm{l})$ of the diluted suspension were inoculated into $1.9 \mathrm{ml}$ of BHI, BHI-DF30, or BHI-DF30 together with a specific iron source in plastic culture tubes $(17$ by $100 \mathrm{~mm})$. Cultures were then allowed to grow at $37^{\circ} \mathrm{C}$ in a gyratory water bath shaker $(200 \mathrm{rpm})$, and $100-\mu \mathrm{l}$ samples and appropriate dilutions thereof were plated onto prewarmed BHI plates at hourly intervals for $8 \mathrm{~h}$. Individual colonies on the BHI agar plates were counted after overnight incubation. All growth experiments were repeated twice for a total of three experiments.

Binding assays with transferrin and lactoferrin. Human transferrin and lactoferrin were conjugated to horseradish peroxidase (HRP) with an ActiZyme-HRP kit (Zymed Laboratories, South San Francisco, Calif.). Cultures $(10 \mathrm{ml}$ of broth in a 500-ml sidearm flask) of strains $035 \mathrm{E}$ and $035 \mathrm{E}-\mathrm{CopB}^{-}$were allowed to grow to a reading of 200 Klett units in BHI or BHI-DF30 as described above. These cells were harvested by centrifugation for $10 \mathrm{~min}$ at $8,000 \times \mathrm{g}$, and each cell pellet was suspended in $1 \mathrm{ml}$ of phosphate-buffered saline, $\mathrm{pH} 7.4$ (PBS). Portions (2 $\mu \mathrm{l})$ were spotted onto Whatman no. 40 filter paper (Whatman, Inc., Clifton, N.J.) and dried at $37^{\circ} \mathrm{C}$ for $1 \mathrm{~h}$. After blocking for $1 \mathrm{~h}$ with Tris-buffered saline, $\mathrm{pH} 7.4$, containing $0.5 \%$ (wt/vol) powdered milk, filter papers were exposed to HRPtransferrin or HRP-lactoferrin $(2 \mu \mathrm{g} / \mathrm{ml})$. After $4 \mathrm{~h}$ of incubation at room temperature, filters were washed three times with Tris-buffered saline and developed with 4-chloro-1-naphthol and $\mathrm{H}_{2} \mathrm{O}_{2}$ in Tris-buffered saline. E. coli $\mathrm{HB} 101$ was used as the negative control strain.

Characterization of $\boldsymbol{M}$. catarrhalis outer membrane proteins. Outer membrane vesicles were prepared by the method described by Murphy and Loeb (34), with M. catarrhalis cells grown overnight in BHI and BHI-DF30. Proteins present in these outer membrane vesicles were resolved by sodium dodecyl sulfate-polyacrylamide gel electrophoresis (SDS-PAGE) and detected either by staining with Coomassie blue or by Western blot (immunoblot) analysis as described previously (23).

Monoclonal antibodies (MAbs). MAb 10F3 is a murine immunoglobulin G2a antibody reactive with the CopB outer membrane protein of $M$. catarrhalis $035 \mathrm{E}$, 012E $(23,24)$, and ATCC 25240. This MAb was used in the form of hybridoma culture supernatant fluid in Western blot analysis.

Disk feeding assays. After overnight growth on BHI agar plates, bacterial colonies were suspended in PBS and cell densities were adjusted to a reading of 100 Klett units. This suspension was diluted 1:100 in BHI, and $100-\mu l$ portions were spread onto BHI-DF30 plates (for $M$. catarrhalis) or BHI plates supplemented with NAD $(10 \mu \mathrm{g} / \mathrm{ml})$ (for $H$. influenzae). Sterile filter paper disks (4-mm diameter) were placed on the agar surface and impregnated with $5 \mu$ of the following iron sources: human transferrin $(10 \mathrm{mg} / \mathrm{ml})$, human lactoferrin $(10$ $\mathrm{mg} / \mathrm{ml})$, human hemoglobin $(1 \mathrm{mg} / \mathrm{ml})$, hemoglobin:haptoglobin $(0.5 \mathrm{mg} / \mathrm{ml})$, bovine hemin $(2.5 \mathrm{mg} / \mathrm{ml})$, heme:hemopexin $(0.5 \mathrm{mg} / \mathrm{ml})$, and ferric citrate $(100$ $\mu \mathrm{M})$. After overnight incubation, the diameter of the zone of bacterial growth around each disk was measured.

${ }^{55} \mathrm{Fe}$ uptake assay. $M$. catarrhalis $035 \mathrm{E}$ and $035 \mathrm{E}-\mathrm{CopB}^{-}$were grown overnight on BHI agar plates, suspended in CDM (46) to a final reading of 20 Klett units, and grown with shaking at $37^{\circ} \mathrm{C}$ in a $95 \%$ air $-5 \% \mathrm{CO}_{2}$ atmosphere to induce iron starvation. These cells were harvested in the late logarithmic phase of growth, and iron uptake assays were performed exactly as previously reported (8) with the following modifications. Nitrocellulose filters were blocked prior to use with $30 \mu \mathrm{l}$ of bovine serum albumin $(10 \mathrm{mg} / \mathrm{ml})$ in CDM. Assays were performed in $200-\mu l$ volumes in 96-well filtration plates (MAHV; Millipore, Bedford, Mass.) at $37^{\circ} \mathrm{C}$ in a $95 \%$ air $-5 \% \mathrm{CO}_{2}$ atmosphere. The vacuum manifold was from the Millipore Multiscreen Assay System. Potassium cyanide (KCN) was dissolved in $1 \times$ CDM. Fifteen percent iron-saturated transferrin was used at a final concentration of $1.08 \mu \mathrm{M}, 22 \%$ iron-saturated lactoferrin was used at $1.28 \mu \mathrm{M}$, and ferric citrate was used at $25 \mu \mathrm{M}$. Membranes were air dried overnight, and the Millipore punch kit was used to separate and collect individual filters for subsequent measurement of bound radioactivity. Data were expressed as counts per minute per microgram of protein. Data were compiled from three individual experiments performed in triplicate on separate days.

\section{RESULTS}

Homology of CopB with TonB-dependent outer membrane proteins. Analysis of the amino acid sequence of CopB from strain $035 \mathrm{E}$ (23) revealed that this $M$. catarrhalis outer membrane protein shared significant homology with the family of TonB-dependent outer membrane proteins. As displayed in Fig. 1, all seven regions of homology, including the TonB box (28) located near the $\mathrm{N}$ terminus, are well conserved in CopB. The highest degree of homology was seen with the iron-regulated FrpB protein of $N$. gonorrhoeae, which was recently described as being homologous to TonB-dependent outer membrane proteins $(6,39)$. As noted by Beucher and Sparling (6),

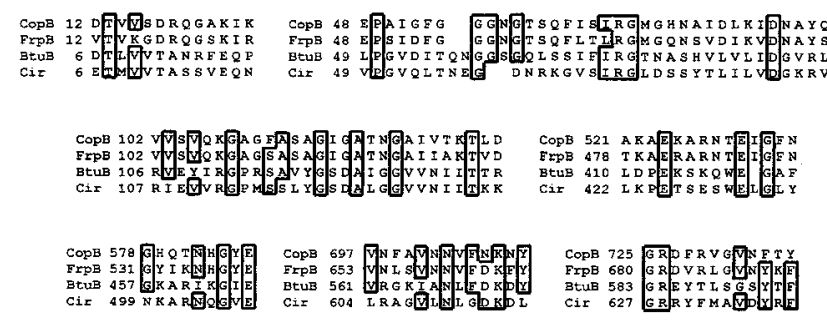

FIG. 1. Peptide alignments between TonB-dependent outer membrane proteins of other organisms and CopB. The other TonB-dependent proteins include FrpB (N. gonorrhoeae iron-regulated outer membrane protein [6]), BtuB (E. coli vitamin $\mathrm{B}_{12}$ receptor [21]), and Cir (E. coli colicin I receptor [35]). The numbers indicate the residue position in the mature protein. The boxes indicates residues that are identical in at least three of the four sequences. 
overall identity and similarity between CopB and this gonococcal outer membrane protein were 52 and $71 \%$, respectively. Similarly, CopB proved to be $49 \%$ identical to the FrpB protein of N. meningitidis (39).

Effect of iron on expression of CopB. The finding that CopB was most similar to the iron-regulated FrpB protein of pathogenic Neisseria species raised the possibility that CopB expression in $M$. catarrhalis might be controlled by iron availability. Moreover, a recent report suggested that CopB expression was increased under conditions of iron limitation (10). To investigate this further, we grew three different wild-type $M$. catarrhalis strains (035E, 012E, and ATCC 25240) as well as the isogenic $\operatorname{cop} B$ mutant strain $035 \mathrm{E}-\mathrm{CopB}^{-}$in both iron-deficient (i.e., BHI-DF30) and iron-replete (i.e., BHI) media and examined the outer membrane proteins present in these cells. Coomassie blue staining of outer membrane proteins resolved by SDS-PAGE indicated that iron limitation in the growth medium did not result in a dramatic increase in expression of outer membrane proteins in two of the three wild-type $M$. catarrhalis strains tested. In both strains 035E (Fig. 2, panel 1, compare lanes A and B) and 012E (Fig. 2, panel 1, compare lanes $\mathrm{G}$ and $\mathrm{H}$ ), iron limitation resulted in a modest increase in expression of a protein with an apparent molecular mass of approximately $80 \mathrm{kDa}$. In strain 012E (Fig. 2, panel 1, lane H), iron limitation also increased expression of one or more proteins with an apparent molecular mass of 90 to $100 \mathrm{kDa}$. In contrast, in strain ATCC 25240 (Fig. 2, panel 1, compare lanes $\mathrm{E}$ and $\mathrm{F}$ ), iron limitation resulted in greatly increased expression of several proteins with apparent molecular masses ranging from 80 to $110 \mathrm{kDa}$. Interestingly, when the isogenic copB mutant was subjected to the same iron limitation, increased expression of several outer membrane proteins with apparent molecular masses of 83 to $100 \mathrm{kDa}$ was observed (Fig. 2, panel 1, compare lanes C and D).

Western blot analysis using MAb 10F3 to detect the CopB protein revealed that $\mathrm{CopB}$ was the $80-\mathrm{kDa}$ protein whose expression was increased only slightly by iron limitation in strains 035E (Fig. 2, panel 2, compare lanes A and B) and 012E (Fig. 2, panel 2, compare lanes $\mathrm{G}$ and H). Similarly, one of the several proteins whose expression was greatly increased by iron limitation in strain ATCC 25240 was CopB (Fig. 2, panel 2, compare lanes $\mathrm{E}$ and $\mathrm{F}$ ). As expected, none of the proteins expressed by the isogenic $\operatorname{cop} B$ mutant reacted with the CopBspecific MAb 10F3 (Fig. 2, panel 2, lanes C and D).

Utilization of iron sources by wild-type and mutant strains of $M$. catarrhalis. The fact that increased expression of CopB was correlated with iron limitation in the growth medium prompted us to investigate whether CopB might be involved in the utilization of iron by $M$. catarrhalis. As a first step, we assessed the ability of $M$. catarrhalis strains to utilize various iron sources for growth in vitro. These experiments involved plating $M$. catarrhalis cells on agar-based media in which iron availability had been restricted by the use of an iron chelator (i.e., Desferal); filter paper disks impregnated with various iron sources were then placed on the surface of the agar, and the resultant growth zones around the disks were recorded.

All three wild-type M. catarrhalis strains tested in this study could utilize ferric citrate, heme, transferrin, and lactoferrin as iron sources (Fig. 3 and data not shown). These same strains did not grow detectably around disks containing hemoglobin, heme:hemopexin, and hemoglobin:haptoglobin. Control experiments involving the wild-type $H$. influenzae type b strain DL42 indicated that sufficient, utilizable quantities of these protein-bound forms of heme were present in these disks (data not shown). Both the wild-type parent strain 035E and its isogenic $\operatorname{cop} B$ mutant grew to the same extent when supplied

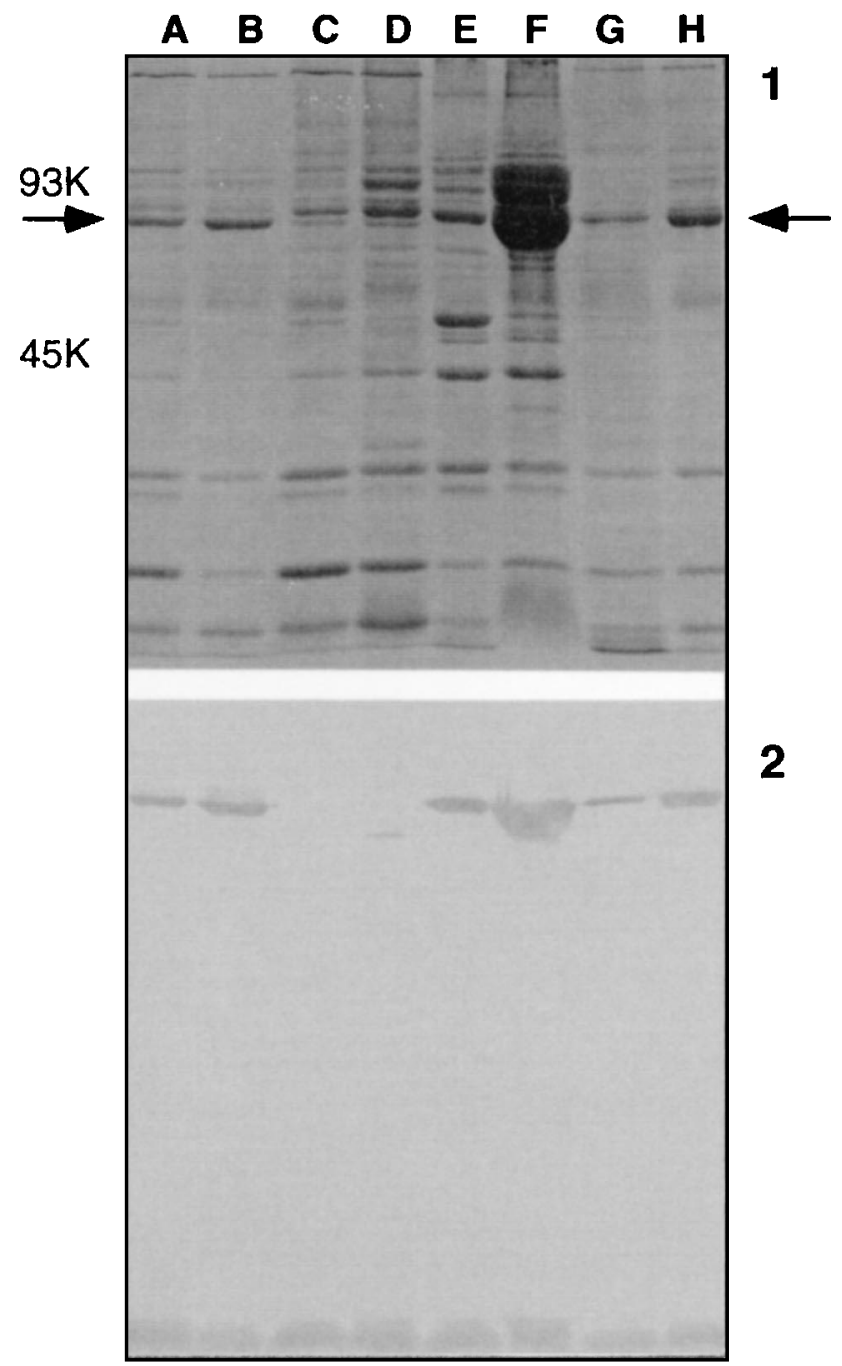

FIG. 2. Expression of outer membrane proteins by wild-type and mutant strains of $M$. catarrhalis grown under iron-replete and iron-limited conditions. Cells of the wild-type strain 035E (lanes A and B), the $\operatorname{copB}$ mutant strain 035E-CopB ${ }^{-}$(lanes C and D), the wild-type strain ATCC 25240 (lanes E and F), and the wild-type strain 012E (lanes $\mathrm{G}$ and $\mathrm{H}$ ) were grown in BHI broth (lanes A, C, E, and G) or in BHI broth containing $30 \mu \mathrm{M}$ Desferal (lanes B, D, F, and $\mathrm{H})$. Proteins present in outer membrane vesicles extracted from these cells were resolved by SDS-PAGE and stained with Coomassie blue (panel 1) or were transferred to nitrocellulose and probed in Western blot analysis with the CopBspecific MAb 10F3 (panel 2). Size markers (in kilodaltons) are present on the left side of this figure. The arrows indicate the position of the CopB protein.

with ferric citrate or heme. In contrast, when either transferrin or lactoferrin was provided as the sole source of iron for growth, the $\operatorname{cop} B$ mutant did not form a visible zone of growth around disks containing either of these iron-containing proteins (Fig. 3). Identical results were obtained when a second, independently constructed $\operatorname{cop} B$ mutant derived from strain 012E (24) was tested in this same system (data not shown).

Growth of $M$. catarrhalis $035 \mathrm{E}$ and $035 \mathrm{E}-\mathrm{CopB}^{-}$in broth under iron-restricted conditions. To investigate further the apparent difference between the wild-type strain $035 \mathrm{E}$ and the isogenic $\operatorname{cop} B$ mutant in their utilization of iron bound to transferrin and lactoferrin, we compared the rates of growth of these two strains in a broth-based medium. These two strains were inoculated into BHI-DF30 containing ferric citrate, trans- 


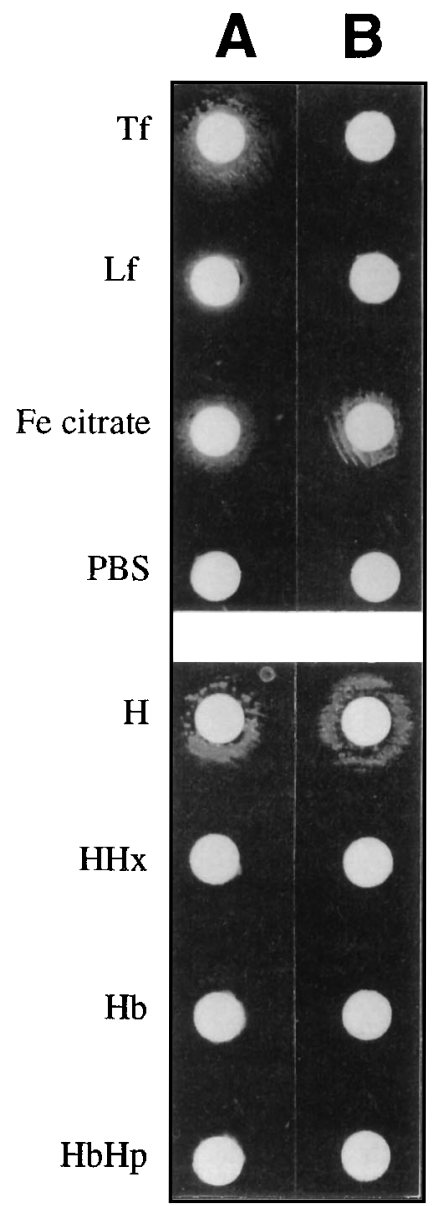

FIG. 3. Utilization of iron sources by wild-type and mutant $M$. catarrhalis strains in a disk feeding assay. The wild-type 035E strain (column A) and its isogenic $\operatorname{copB}$ mutant strain $035 \mathrm{E}-\mathrm{CopB}^{-}$(column B) were plated on $\mathrm{BHI}$ medium containing $30 \mu \mathrm{M}$ Desferal, and then sterile filter paper disks were pressed into the agar surface and spotted with the following iron sources: transferrin (Tf), lactoferrin (Lf), ferric citrate, PBS (negative control), hemin (H), heme:hemopexin $(\mathrm{HHx})$, hemoglobin $(\mathrm{Hb})$, and hemoglobin:haptoglobin $(\mathrm{HbHp})$.

ferrin, or lactoferrin as the iron source, and the extent of growth was measured by viability determinations.

Both the wild-type and mutant strains grew at the same rate in BHI broth (Fig. 4A and B, closed and open circles, respectively). The addition of the iron chelator Desferal at a final concentration of $30 \mu \mathrm{M}$ effectively stopped growth of both the wild-type and mutant strains (Fig. 4A and B, closed and open squares, respectively). Addition of $25 \mu \mathrm{M}$ ferric citrate overcame completely the growth-inhibiting effect of the Desferal (Fig. 4A and B, closed and open triangles, respectively). Addition of a lower concentration $(5 \mu \mathrm{M})$ of ferric citrate allowed slow growth of both the wild-type and mutant strains at nearly equivalent rates in the presence of Desferal (Fig. 4A and B, closed and open inverted triangles, respectively). These results indicate that lack of CopB expression did not affect significantly the ability of $M$. catarrhalis to obtain iron from ferric citrate.

When either transferrin or lactoferrin was supplied to the wild-type strain in the presence of $30 \mu \mathrm{M}$ Desferal (Fig. 5, closed triangles), this strain grew as rapidly and to the same extent as it did in BHI broth alone (Fig. 5, closed circles). As expected, the $c o p B$ mutant (Fig. 5, open circles) grew as well as the wild-type strain (Fig. 5, closed circles) in BHI broth; nei-

A

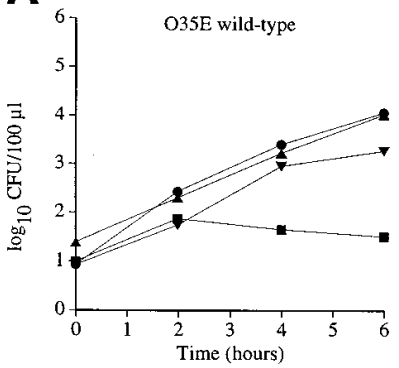

B

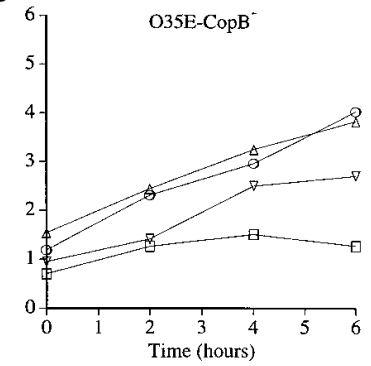

FIG. 4. Growth of wild-type and mutant $M$. catarrhalis strains in iron-replete and iron-limited broth supplemented with varying levels of ferric citrate. The wild-type strain $035 \mathrm{E}(\mathrm{A})$ and the isogenic $\operatorname{copB}$ mutant $035 \mathrm{E}-\mathrm{CopB}^{-}$(B) were inoculated into BHI broth (circles) and into BHI broth containing $30 \mu \mathrm{M}$ Desferal alone (squares) or Desferal and either $5 \mu \mathrm{M}$ (inverted triangles) or $25 \mu \mathrm{M}$ (triangles) ferric citrate. Growth was monitored over time by plating samples of the cultures onto BHI agar plates.

ther strain grew in BHI containing $30 \mu \mathrm{M}$ Desferal (Fig. 5, closed and open squares). When transferrin was the sole iron source available to the $\operatorname{cop} B$ mutant (Fig. 5A, open triangles), this mutant strain did not grow and the number of viable organisms decreased to a level beneath that of the starting inoculum. Similarly, when the $\operatorname{cop} B$ mutant was supplied with lactoferrin as the iron source (Fig. 5B, open triangles), it did not grow as rapidly as did the wild-type parent strain (Fig. 5B, closed triangles), although the number of viable organisms did not decrease with time (as was the case with transferrin).

Binding of transferrin and lactoferrin by wild-type and mutant $M$. catarrhalis cells. The broth-based growth experiments described above confirmed that the isogenic $\operatorname{cop} B$ mutant was less able than its wild-type parent strain to utilize both transferrin and lactoferrin as sources of iron. To determine whether the decreased utilization of these iron sources was the result of a significant diminution in the ability of the $\operatorname{cop} B$ mutant to bind these two proteins, a dot blot assay was used to measure binding of both of these iron carrier proteins to $M$. catarrhalis cells. The wild-type and mutant strains were grown in both BHI and BHI-DF30 and tested for their ability to bind HRPconjugated forms of transferrin and lactoferrin (Fig. 6). There was no apparent difference in the relative abilities of the wildtype strain (Fig. 6, column A) and mutant strain (Fig. 6, column B) to bind transferrin, regardless of whether the cells were

A

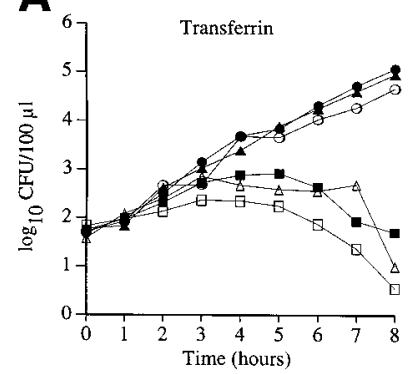

FIG. 5. Growth of wild-type and mutant M. catarrhalis strains in iron-replete or iron-limited broth supplemented with either transferrin or lactoferrin. (A) The wild-type strain $035 \mathrm{E}$ (closed symbols) and the isogenic $\operatorname{copB}$ mutant (open symbols) were inoculated into BHI broth alone (circles), BHI containing $30 \mu \mathrm{M}$ Desferal (squares), and BHI containing $30 \mu \mathrm{M}$ Desferal and $10 \mu \mathrm{M}$ transferrin (triangles). (B) The same experiment was repeated except that $2.5 \mu \mathrm{M}$ lactoferrin was substituted for transferrin. Growth was monitored as described in the legend for Fig. 4. 


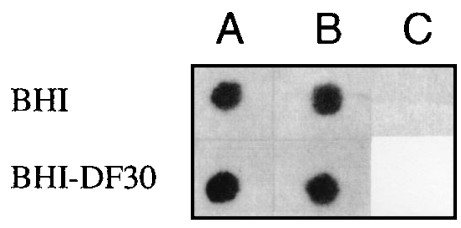

HRP-Tf

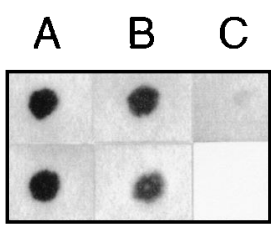

HRP-Lf
FIG. 6. Binding of transferrin and lactoferrin by wild-type and mutant strains of $M$. catarrhalis. Cells of the wild-type strain 035E (column A) and the $\operatorname{cop} B$ mutant strain 035E-CopB ${ }^{-}$(column B) were grown in $\mathrm{BHI}$ or in $\mathrm{BHI}$ containing $30 \mu \mathrm{M}$ Desferal (BHI-DF30), harvested by centrifugation, and spotted onto filter paper for incubation with HRP-coupled human transferrin (HRP-Tf) and lactoferrin (HRP-Lf). E. coli HB101 grown on Luria-Bertani agar (column C) was included as a negative control.

grown under iron-limited (BHI-DF30) or iron-replete (BHI) conditions. When tested for lactoferrin binding, the $\operatorname{cop} B$ mutant (Fig. 6, column B) appeared to bind slightly less lactoferrin than did the wild-type parent strain (Fig. 6, column A). This difference, though slight, was highly reproducible. Testing of serial twofold dilutions of these cell suspensions in this dot blot assay did not reveal any differences in transferrin-binding ability between the wild-type and mutant strains and confirmed that the mutant bound slightly less lactoferrin than did the wild-type strain (data not shown).

The fact that iron limitation did not result in an appreciable increase in the binding of either lactoferrin or transferrin by the wild-type $M$. catarrhalis strain $035 \mathrm{E}$ correlated with the very modest increase in expression of strain $035 \mathrm{E}$ outer membrane proteins in response to iron limitation (Fig. 2, panel 1, lanes A and B). This observation also confirms the findings of Schryvers and Lee (44), who demonstrated that, in comparison with neisserial species, iron starvation resulted in only a moderate increase in the binding of transferrin and lactoferrin by a wildtype $M$. catarrhalis strain.

Uptake of ${ }^{55} \mathrm{Fe}$ by wild-type and mutant $M$. catarrhalis strains. A well-described iron uptake assay system $(8,16)$ was used to determine whether the $\operatorname{cop} B$ mutant differed from its parent strain in its ability to take up iron from transferrin, lactoferrin, and ferric citrate. Controls included the use of a wild-type strain of N. gonorrhoeae (FA19) as well as a N. gonorrhoeae tbpA mutant (strain FA6747) (12) and a N. gonorrhoeae lbp mutant (strain FA6775). Both the wild-type M. catarrhalis strain and the wild-type $N$. gonorrhoeae strain took up ${ }^{55} \mathrm{Fe}$ from all three test compounds at equivalent rates (data not shown). As expected, the $N$. gonorrhoeae tbpA mutant did not take up iron from transferrin (Fig. 7C) and the $N$. gonorrhoeae lbp $A$ mutant did not take up iron from lactoferrin (Fig. 7B). Iron acquisition by the $M$. catarrhalis $\operatorname{copB}$ mutant (Fig. 7E) from transferrin and lactoferrin was substantially reduced relative to the wild-type parent strain (Fig. 7D). In contrast, uptake of radiolabeled iron from ferric citrate by the $\operatorname{cop} B \mathrm{mu}-$ tant (Fig. 7E) proceeded almost as rapidly as it did in the wildtype parent strain (Fig. 7D).

\section{DISCUSSION}

TonB-dependent outer membrane proteins, the proper functioning of which depends on the presence of a functional TonB protein, bind various ligands (e.g., iron-siderophore complexes, vitamin $B_{12}$, heme) for subsequent transport into the gramnegative bacterial cell $(5,19,25,26,41)$. The TonB protein itself spans the periplasm and is thought to be necessary for energy transduction from the cytoplasmic membrane to ener- gy-requiring outer membrane processes $(12,26,41)$. These TonB-dependent outer membrane proteins have seven regions of homology, as defined by Lundrigan and Kadner (28), including the TonB box located near the $\mathrm{N}$ terminus, which has been implicated in direct interaction between TonB and these outer membrane proteins $(5,9,22,43)$.

Close inspection of the amino acid sequence of CopB revealed that it possesses not only this TonB box near the $\mathrm{N}$ terminus but also the other six regions of homology. This similarity was missed because of a database searching error in a previous study (23). The last region of homology between these TonB-dependent proteins and CopB is at the C-terminal end of the molecule, where an amphipathic motif terminates with an aromatic amino acid residue. This is characteristic of virtually all outer membrane proteins and has been suggested to be essential for outer membrane localization (45).

CopB is expressed in abundance when M. catarrhalis is grown in complex media (e.g., BHI broth) $(23,34)$. Therefore, the finding that $\mathrm{CopB}$ is homologous to TonB-dependent outer membrane proteins was somewhat surprising inasmuch as most previously described TonB-dependent proteins are not constitutively expressed in other bacteria but are instead produced in response to environmental conditions (e.g., iron starvation, heme limitation, etc.). However, a recent report raised the possibility that CopB expression could be regulated to some extent by the availability of iron (10). The results of the present study confirmed those of this previous report (10) and also revealed considerable, strain-dependent variation in the level of expression of CopB in response to iron limitation (Fig. 2).

Interestingly, CopB proved to be most similar (49 to $52 \%$ identity) to the iron-regulated $\operatorname{FrpB}$ protein expressed by pathogenic Neisseria species $(6,39)$. This finding, together with the ability of iron availability to affect CopB expression (Fig. 2), raised the possibility that CopB might be involved in iron utilization by $M$. catarrhalis. We first tested several wild-type

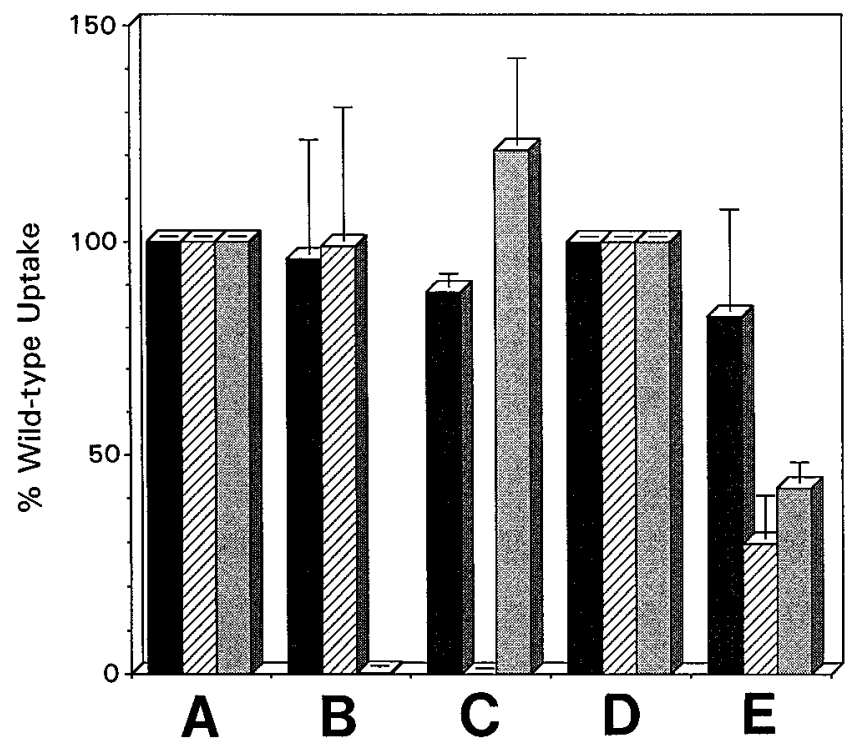

FIG. 7. Uptake of ${ }^{55} \mathrm{Fe}$ from ${ }^{55} \mathrm{Fe}$-transferrin, ${ }^{55} \mathrm{Fe}$-lactoferrin, and ${ }^{55} \mathrm{Fe}-$ citrate by wild-type and mutant strains of both $M$. catarrhalis and $N$. gonorrhoeae. Uptake of ${ }^{55} \mathrm{Fe}$ from ferric citrate (black columns), transferrin (hatched columns), and lactoferrin (stippled columns) was measured as described in Materials and Methods. (A) Wild-type N. gonorrhoeae strain FA19; (B) N. gonorrhoeae lbpA mutant strain FA6775; (C) N. gonorrhoeae tbpA mutant strain FA6747; (D) wild-type $M$. catarrhalis strain 035E; (E) M. catarrhalis copB mutant strain $035 \mathrm{E}-\mathrm{CopB} \mathrm{B}^{-} .{ }^{55} \mathrm{Fe}$ uptake by the wild-type parent strains was set at $100 \%$ for comparison with the mutants. Standard deviations are indicated by error bars. 
M. catarrhalis isolates for their ability to utilize ferric citrate, heme, hemoglobin, hemoglobin:haptoglobin, heme:hemopexin, transferrin, and lactoferrin as sources of iron. All wild-type isolates tested could use only iron citrate, heme, lactoferrin, and transferrin for growth. By both disk feeding experiments (Fig. 3) and broth-based growth determinations (Fig. 4 and 5), it was found that an isogenic $\operatorname{cop} B$ mutant was markedly impaired in its ability to utilize lactoferrin and transferrin but not iron citrate.

Binding of lactoferrin and transferrin by the wild-type strains and that by $\operatorname{cop} B$ mutant strains were essentially equivalent (Fig. 6), indicating that the $\operatorname{cop} B$ mutation did not affect binding of these iron sources. This finding eliminated the possibility that CopB is a homolog of either the Tbp1 or Lbp receptor described elsewhere for pathogenic Neisseria species (7, 12, 27 , 38 ). While the molecular mass of CopB is similar to that of Tbp2, the latter has been shown to be a lipoprotein with no similarity to TonB-dependent outer membrane proteins $(2$, 27).

Radiolabeled iron uptake studies revealed that the $\operatorname{copB}$ mutant was impaired in its uptake of ${ }^{55} \mathrm{Fe}$ from both lactoferrin and transferrin, but not from ferric citrate (Fig. 7). The fact that lack of CopB expression adversely affects but does not eliminate the uptake of iron from both transferrin and lactoferrin indicates that this outer membrane protein may facilitate the extraction of iron from both of these carrier proteins. Similarly, CopB may somehow stimulate the transport of transferrin- or lactoferrin-derived iron across the outer membrane. Alternatively, the fact that utilization and uptake of both lactoferrin-bound and transferrin-bound iron were affected to approximately the same extent in the $\operatorname{cop} B$ mutant (Fig. 7) raised the possibility that the effect of the $\operatorname{cop} B$ mutation on these processes might be indirect and caused by a concomitant change in outer membrane architecture that affects the ability of the $M$. catarrhalis lactoferrin- and transferrin-binding proteins $(44,47)$ to extract iron from their respective ligands.

With regard to the latter possibility, a similar situation has been reported with the iron-regulated FrpB protein (which is $52 \%$ identical to CopB) of $N$. gonorrhoeae, in which a frpB mutation affected the uptake of iron from heme in a nonspecific manner (6). An isogenic frpB mutant of $N$. gonorrhoeae was able to use transferrin, lactoferrin, ferric citrate, hemoglobin, and hemoglobin-haptoglobin complexes as iron sources but was reduced by $60 \%$ in its ability to use heme-bound iron compared with the wild-type parent strain. This effect of the $\operatorname{frp} B$ mutation appeared to be nonspecific, however, as hemebound iron uptake by isogenic gonococcal mutants lacking Tbp1, Tbp2, or Lbp was similarly affected (6). It has also been reported that an isogenic meningococcal $\operatorname{frp} B$ mutant was unaffected in its ability to use iron bound to transferrin or lactoferrin (39).

It is of interest that the phenotype of the $\operatorname{cop} B$ mutant, with regard to the utilization of iron bound to transferrin and lactoferrin, resembles that of one type of gonococcal iron uptake mutant described several years ago (17). The pleiotropic tlu mutants of $N$. gonorrhoeae were markedly impaired in their ability to use iron from transferrin and lactoferrin, while iron utilization from ferric citrate, heme, and hemoglobin was unaffected (17). These $t l u$ mutants were obtained by streptonigrin-based enrichment after chemical mutagenesis, and the affected genetic locus was found not to be linked with either transferrin or lactoferrin utilization loci (36).

It must be noted that CopB has been shown to bind antibodies that enhance pulmonary clearance of $M$. catarrhalis (23). In this regard, CopB is again similar to the FrpB protein of the meningococcus, which has also been shown to be a target for biologically active antibodies $(37,39)$. In addition, lack of CopB expression affects the resistance of $M$. catarrhalis to killing by normal human serum and the ability of this organism to resist clearance from the lungs of mice (24). While it is very likely that the latter in vivo survival phenotype is directly related to the serum sensitivity issue, when taken together with the results of the present study, it is clear that a $\operatorname{cop} B$ mutation exerts significant pleiotropic effects on $M$. catarrhalis. If CopB expression is truly essential for survival of this pathogen in vivo, this would bode well for further consideration of CopB as a potential vaccine candidate.

\section{ACKNOWLEDGMENTS}

This study is based in part upon work supported by the Texas Advanced Technology Program under grant no. 003660-057. C.A. was supported by a grant from the National Research Foundation of Switzerland.

\section{REFERENCES}

1. Alexander, H. E. 1965. The Haemophilus group, p. 724-741. In R. J. Dubos and J. G. Hirsch (ed.), Bacterial and mycotic infections of man. J. B. Lippincott Co., Philadelphia.

2. Anderson, J. E., P. F. Sparling, and C. N. Cornelissen. 1994. Gonococcal transferrin-binding protein 2 facilitates but is not essential for transferrin utilization. J. Bacteriol. 176:3162-3170.

3. Aspin, M. M., A. Hoberman, J. McCarty, S. E. McLinn, S. Aronoff, D. J. Lang, and A. Arrieta. 1994. Comparative study of the safety and efficacy of clarithromycin and amoxicillin-clavulanate in the treatment of acute otitis media in children. J. Pediatr. 125:135-141.

4. Bartos, L. C., and T. F. Murphy. 1988. Comparison of the outer membrane proteins of 50 strains of Branhamella catarrhalis. J. Infect. Dis. 158:761-765.

5. Bell, P. E., C. D. Nau, J. T. Brown, J. Konisky, and R. J. Kadner. 1990 Genetic suppression demonstrates interaction of TonB protein with outer membrane proteins in Escherichia coli. J. Bacteriol. 172:3826-3829.

6. Beucher, M., and P. F. Sparling. 1995. Cloning, sequencing, and characterization of the gene encoding FrpB, a major iron-regulated, outer membrane protein of Neisseria gonorrhoeae. J. Bacteriol. 177:2041-2049.

7. Biswas, G. D., and P. F. Sparling. 1995. Characterization of $l b p A$, the structural gene for a lactoferrin receptor in Neisseria gonorrhoeae. Infect. Immun. 63:2958-2967.

8. Blanton, K. J., G. D. Biswas, J. Tsai, J. Adams, D. W. Dyer, S. M. Davis, G. G. Koch, P. K. Sen, and P. F. Sparling. 1990. Genetic evidence that Neisseria gonorrhoeae produces specific receptors for transferrin and lactoferrin. J. Bacteriol. 172:5225-5235

9. Brewer, S., M. Tolley, I. P. Trayer, G. C. Barr, C. J. Dorman, K. Hannavy, C. F. Higgins, J. S. Evans, B. A. Levine, and M. R. Wormald. 1990. Structure and function of X-Pro dipeptide repeats in the TonB proteins of Salmonella typhimurium and Escherichia coli. J. Mol. Biol. 216:883-895.

10. Campagnari, A. A., K. L. Shanks, and D. W. Dyer. 1994. Growth of Moraxella catarrhalis with human transferrin and lactoferrin: expression of iron-repressible proteins without siderophore production. Infect. Immun. 62:49094914.

11. Cope, L. D., R. Yogev, U. Muller-Eberhard, and E. J. Hansen. 1995. A gene cluster involved in the utilization of both free heme and heme:hemopexin by Haemophilus influenzae type b. J. Bacteriol. 177:2644-2653.

12. Cornelissen, C. N., G. D. Biswas, J. Tsai, D. K. Paruchuri, S. A. Thompson and P. F. Sparling. 1992. Gonococcal transferrin-binding protein 1 is required for transferrin utilization and is homologous to TonB-dependent outer membrane receptors. J. Bacteriol. 174:5788-5797.

13. Davies, B. I., and F. P. V. Maesen. 1988. The epidemiology of respiratory tract pathogens in Southern Netherlands. Eur. Respir. J. 1:415-420.

14. Del Beccaro, M. A., P. M. Mendelman, A. F. Inglis, M. A. Richardson, N. O Duncan, C. R. Clausen, and T. L. Stull. 1992. Bacteriology of acute otitis media: a new perspective. J. Pediatr. 120:81-84.

15. Doern, G. V., M. J. Miller, and R. E. Winn. 1981. Branhamella (Neisseria) catarrhalis systemic disease in humans. Arch. Intern. Med. 141:1690-1692.

16. Dyer, D. W., W. McKenna, J. P. Woods, and P. F. Sparling. 1987. Isolation by streptonigrin enrichment and characterization of a transferrin-specific iron uptake mutant of Neisseria meningitidis. Microb. Pathog. 3:351-363.

17. Dyer, D. W., E. P. West, W. McKenna, S. A. Thompson, and P. F. Sparling. 1988. A pleiotropic iron-uptake mutant of Neisseria meningitidis lacks a 70-kilodalton iron-regulated protein. Infect. Immun. 56:977-983.

18. Gan, V. N., H. Kusmiesz, S. Shelton, and J. D. Nelson. 1991. Comparative evaluation of loracarbef and amoxicillin-clavulanate for acute otitis media. Antimicrob. Agents Chemother. 35:967-971.

19. Gudmundsdottir, A., P. E. Bell, M. D. Lundrigan, C. Bradbeer, and R. J. Kadner. 1989. Point mutations in a conserved region (TonB box) of Esche- 
richia coli outer membrane protein $\mathrm{BtuB}$ affect vitamin $\mathrm{B}_{12}$ transport. J. Bacteriol. 171:6526-6533.

20. Hager, H., A. Verghese, S. Alvarez, and S. L. Berk. 1987. Branhamella catarrhalis respiratory infections. Rev. Infect. Dis. 9:1140-1149.

21. Heller, K. J., and R. J. Kadner. 1985. Nucleotide sequence of the gene for the vitamin $\mathrm{B}_{12}$ receptor protein in the outer membrane of Escherichia coli. J. Bacteriol. 161:904-908.

22. Heller, K. J., R. J. Kadner, and K. Gunter. 1988. Suppression of btuB451 mutation by mutations in the ton $B$ gene suggests a direct interaction between TonB and TonB-dependent receptor proteins in the outer membrane of Escherichia coli. Gene 64:147-153.

23. Helminen, M. E., I. Maciver, J. L. Latimer, L. D. Cope, G. H. McCracken, Jr., and E. J. Hansen. 1993. A major outer membrane protein of Moraxella catarrhalis is a target for antibodies that enhance pulmonary clearance of the pathogen in an animal model. Infect. Immun. 61:2003-2010.

24. Helminen, M. E., I. Maciver, J. L. Latimer, S. R. Lumbley, L. D. Cope, G. H. McCracken, Jr., and E. J. Hansen. 1993. A mutation affecting expression of a major outer membrane protein of Moraxella catarrhalis alters serum resistance and survival of this organism in vivo. J. Infect. Dis. 168:1194-1201.

25. Henderson, D. P., and S. M. Payne. 1994. Characterization of the Vibrio cholerae outer membrane heme transport protein HutA: sequence of the gene, regulation of expression, and homology to the family of TonB-dependent proteins. J. Bacteriol. 176:3269-3277.

26. Kadner, R. J. 1990. Vitamin $B_{12}$ transport in Escherichia coli: energy coupling between membranes. Mol. Microbiol. 4:2027-2033.

27. Legrain, M., V. Mazarin, S. W. Irwin, B. Bouchon, M.-J. Quentin-Millet, and A. B. Schryvers. 1993. Cloning and characterization of Neisseria meningitidis genes encoding the transferrin-binding proteins Tbp1 and Tbp2. Gene 130: $73-80$.

28. Lundrigan, M. D., and R. J. Kadner. 1986. Nucleotide sequence of the gene for the ferrienterochelin receptor FepA in Escherichia coli: homology among outer membrane receptors that interact with TonB. J. Biol. Chem. 261: 10797-10801.

29. Malkamaki, M., E. Honkanen, M. Leinonen, and P. H. Makela. 1983. Branhamella catarrhalis as a cause of bacteremic pneumonia. Scand. J. Infect. Dis. 15:125-126.

30. Maniatis, T., E. F. Fritsch, and J. Sambrook. 1982. Molecular cloning: a laboratory manual. Cold Spring Harbor Laboratory Press, Cold Spring Harbor, N.Y.

31. McLeod, D. T., F. Ahmad, S. Capewell, M. J. Croughan, M. A. Calder, and A. Seaton. 1986. Increase in bronchopulmonary infection due to Branhamella catarrhalis. Br. Med. J. [Clin. Res.] 292:1103-1105.

32. Mickelsen, P. A., and P. F. Sparling. 1981. Ability of Neisseria gonorrhoeae, Neisseria meningitidis, and commensal Neisseria species to obtain iron from transferrin and iron compounds. Infect. Immun. 33:555-564.

33. Murphy, T. F., and L. C. Bartos. 1989. Surface-exposed and antigenically conserved determinants of outer membrane proteins of Branhamella ca- tarrhalis. Infect. Immun. 57:2938-2941.

34. Murphy, T. F., and M. R. Loeb. 1989. Isolation of the outer membrane of Branhamella catarrhalis. Microb. Pathog. 6:159-174.

35. Nau, C. D., and J. Konisky. 1989. Evolutionary relationship between the TonB-dependent outer membrane transport proteins: nucleotide and amino acid sequences of the Escherichia coli colicin I receptor gene. J. Bacteriol. 171:1041-1047.

36. Norn, S., P. Clementsen, A. Fomsgaard, and M. Kilian. 1990. Haemophilus influenzae potentiates basophil histamine release possibly by its endotoxins. Agents Actions 30:57-60.

37. Pettersson, A., B. Kuipers, M. Pelzer, E. Verhagen, R. H. Tiesjema, J. Tommassen, and J. T. Poolman. 1990. Monoclonal antibodies against the 70-kilodalton iron-regulated protein of Neisseria meningitidis are bactericidal and strain specific. Infect. Immun. 58:3036-3041.

38. Pettersson, A., A. Maas, and J. Tommassen. 1994. Identification of the iroA gene product of Neisseria meningitidis as a lactoferrin receptor. J. Bacteriol. 176:1764-1766.

39. Pettersson, A., A. Maas, D. van Wassenaar, P. van der Ley, and J. Tommassen. 1995. Molecular characterization of FrpB, the 70-kilodalton iron-regulated outer membrane protein of Neisseria meningitidis. Infect. Immun. 63: 4181-4184.

40. Post, J. C., R. A. Preston, J. J. Aul, M. Larkins-Pettigrew, J. Rydquist-White, K. W. Anderson, R. M. Wadowsky, D. R. Reagan, E. S. Walker, L. A. Kingsley, A. E. Magit, and G. D. Ehrlich. 1995. Molecular analysis of bacterial pathogens in otitis media with effusion. JAMA 273:1598-1604.

41. Postle, K. 1990. TonB and the Gram-negative dilemma. Mol. Microbiol. 4: 2019-2025.

42. Sambrook, J., E. F. Fritsch, and T. Maniatis. 1989. Molecular cloning: laboratory manual, 2nd ed. Cold Spring Harbor Laboratory Press, Cold Spring Harbor, N.Y.

43. Schoffler, H., and V. Braun. 1989. Transport across the outer membrane of Escherichia coli via the FhuA receptor is regulated by the TonB protein of the cytoplasmic membrane. Mol. Gen. Genet. 217:378-383.

44. Schryvers, A. B., and B. C. Lee. 1989. Comparative analysis of the transferrin and lactoferrin binding proteins in the family Neisseriaceae. Can. J. Microbiol. 35:409-415.

45. Stuyve, M., M. Moons, and J. Tommassen. 1991. Carboxyterminal phenylalanine is essential for the correct assembly of a bacterial outer membrane protein. J. Mol. Biol. 218:141-148.

46. West, S. E., and P. F. Sparling. 1987. Aerobactin utilization by Neisseria gonorrhoeae and cloning of a genomic DNA fragment that complements Escherichia coli fhuB mutations. J. Bacteriol. 169:3414-3421.

47. Yu, R.-H., and A. B. Schryvers. 1993. The interaction between human transferrin and transferrin binding protein 2 from Moraxella (Branhamella) $\mathrm{ca}$ tarrhalis differs from that of other human pathogens. Microb. Pathog. 15: 433-445.

Editor: B. I. Eisenstein 